\title{
MJMR RADIOACTIVE INJECTIONS FOR CHRONIC SWOLLEN LEGS: CASE SERIES OF BILATERAL LYMPHOEDEMA
}

\author{
Ahmad Zaid Zanial \\ Nuclear Medicine Department, Hospital Kuala Lumpur, Jalan Pahang, Kuala Lumpur, Malaysia \\ Corresponding Author E-mail: ahmadzaidx@gmail.com
}

\begin{abstract}
Lymphoedema is a chronic condition due to lymphatic drainage abnormality causing swollen limbs. Although less-known, lymphoscintigraphy or evaluation of lymphatic system following radioactive injections is a relatively simple and valuable functional imaging. This case series report 3 cases of bilateral lower limb lymphoedema in order to highlight the usefulness and complementary diagnostic role of lymphoscintigraphy as well as clinical characteristics and scintigraphy findings of these patients. They were obese, but none had any lower limb trauma or documented deep vein thrombosis. Clinical history and manifestations were not suggestive of filarial infections. Case 1; 61 years old male with bilateral chronic swollen legs and underlying liver cirrhosis demonstrated abnormal tracer progression with bilateral mild diffuse dermal backflow on scintigraphy. Case 2; 43 years old male with a prominent right leg swelling and history of cellulitis demonstrated scintigraphically abnormal tracer progression in right leg and bilateral dermal backflow with popliteal nodes. Case 3; 46 years old male with bilateral chronic swollen legs and history of cellulitis demonstrated abnormal tracer progression in left leg with contralateral right dermal backflow on scintigraphy. All patients had revealed scintigraphy findings suggestive of bilateral lower limb lymphoedema.
\end{abstract}

\section{Keywords: Swollen Legs, Lymphoedema, Lymphoscintigraphy, Radioactive}

\section{INTRODUCTION}

Lymphoedema is defined as fluid accumulation in the tissue arising from impaired lymphatic drainage due to either congenital or acquired anomalies of lymphatic outflow (Szuba, 1998). It is a chronic condition associated with swollen body parts or regions. Lymphoedema is most common in the lower limbs with about $80 \%$ of cases, but can also occur in the arms, face, trunk and external genitalia (Yuan, 2006). In many cases of advanced disease, a typical history and characteristic clinical presentation could establish the diagnosis of lymphoedema with almost certainty.

Nevertheless, additional tests are sometimes necessary to confirm the presence of impaired lymphatic flow and/or the typical pattern of abnormal fluid distribution within tissues of affected limb particularly in early stages or cases with mild swelling (Szuba, 1998). Moreover, some patients may present with lymphoedema of both lower limbs and hence should be differentiate with other systemic aetiologies or venous insufficiency that can pose possible diagnostic challenges (Hennessy, 2017).

Lymphoscintigraphy is a valuable and relatively noninvasive procedure that can be very helpful for diagnosis of lymphoedema. It involves injection of small amount of radioactive particulate into soft tissue of the limb or body part followed by imaging using gamma camera to evaluate the lymphatic system and lymph nodes (Sadeghi, 2010). Hence, this case series report 3 cases of bilateral lower limb lymphoedema in order to highlight the complementary diagnostic role of scintigraphy as well as clinical characteristics and scintigraphy findings of these patients.

\section{CASE SERIES}

All patients were hypertensive and obese with body mass index (BMI) over $30 \mathrm{~kg} / \mathrm{m} 2$, but none had any lower limb trauma or documented deep vein thrombosis. Clinical history and manifestations were not suggestive of filarial infections. Baseline and preliminary investigations were unremarkable. They were referred for further evaluation and underwent 
lower limb lymphoscintigraphy at the Nuclear Medicine Department, Hospital Kuala Lumpur between December 2015 and July 2017.

They received subcutaneous injections of radioactive Technetium99m-nanocolloid tracer at the first and second web-spaces of each foot totalling 2 millicurie. Based on our standard departmental protocol, inoculation sites were gently massage for approximately 2 minutes prior to a 15-minute dynamic imaging acquisition. Subsequent, immediate phase and delayed phase whole-body scanning done between 1 and 4 hours after injections. None of them developed any adverse effect or complications related to the procedure.

Case 1; 61 years old male with bilateral chronic swollen legs for 24 months and underlying liver cirrhosis demonstrated abnormal tracer progression with bilateral mild diffuse dermal backflow on scintigraphy (Figure 1).

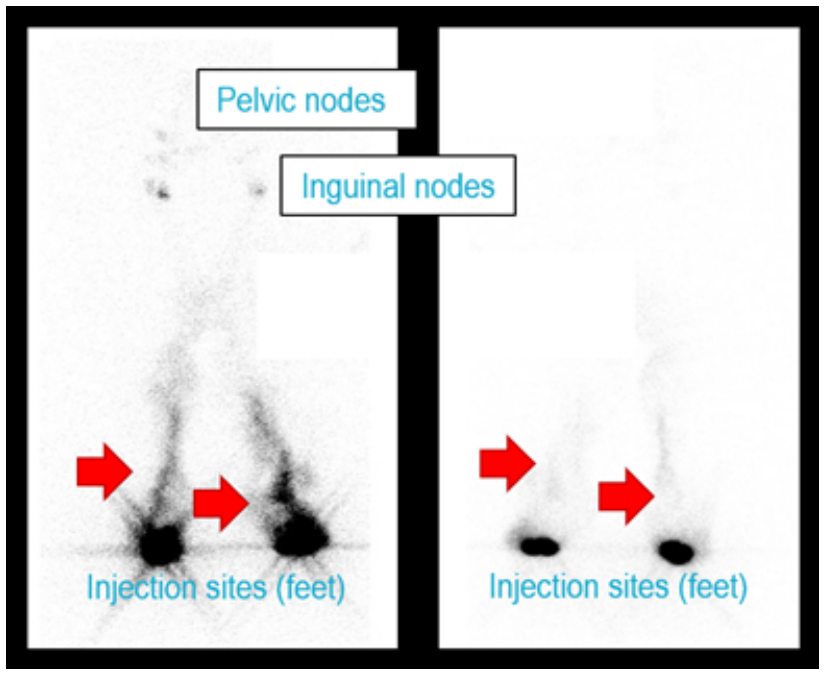

Figure 1: (Left-anterior and Right-posterior): Abnormal tracer progression from injection sites associated with reduced tracer activity at the inguinal and pelvic nodes (left side appears to be more affected). Bilateral mild diffuse dermal backflow (red arrow) noted.

Case 2; 43 years old male with a prominent right leg swelling for 6 months and history of cellulitis demonstrated scintigraphically abnormal tracer progression in right leg and bilateral dermal backflow with popliteal nodes (Figure 2).

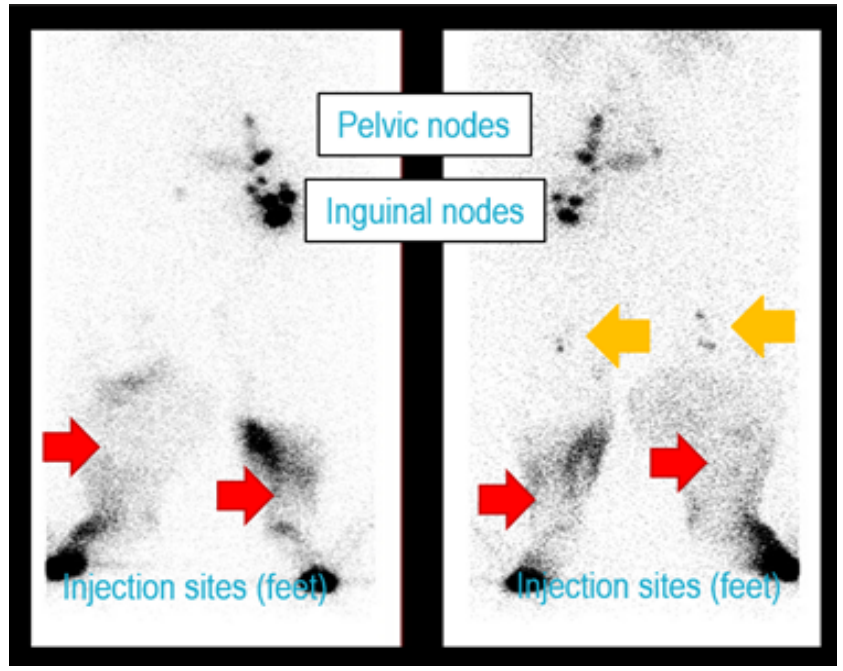

Figure 2: (Left-anterior and Right-posterior): Abnormal tracer progression at right lower limb with poor visualisation of right inguinal and pelvic nodes. Bilateral prominent dermal backflow (red arrow) and popliteal nodes (yellow arrow) noted.

Case 3; 46 years old male with bilateral chronic swollen legs for 24 months and history of cellulitis demonstrated abnormal tracer progression in left leg with contralateral right dermal backflow on scintigraphy (Figure 3).

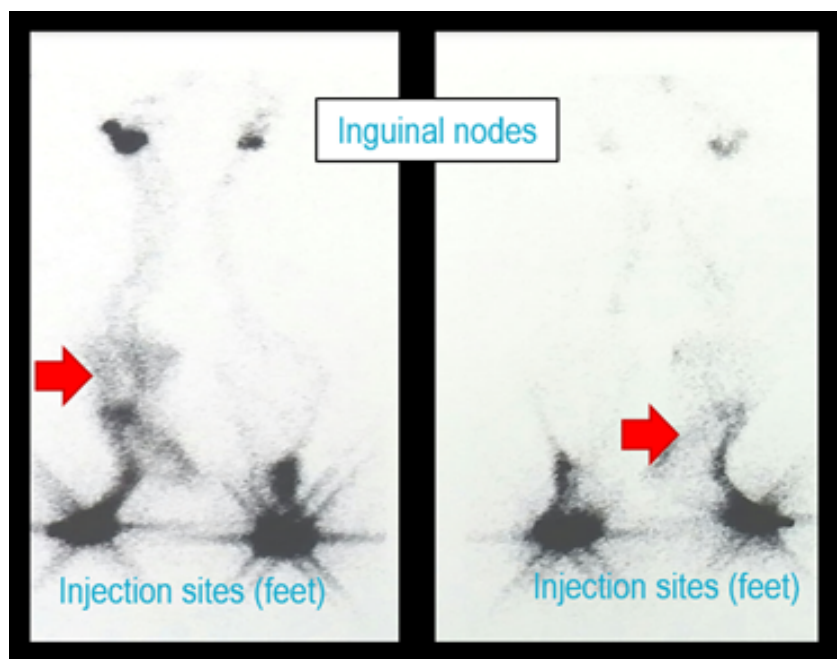

Figure 3: (Left-anterior and Right-posterior): Abnormal tracer progression at left lower limb with relatively reduced activity at left inguinal nodes. Contralateral prominent dermal backflow (red arrow) at right leg noted. 


\section{DISCUSSION}

Lymphoedema can be classified into primary and secondary causes. Primary lymphoedema include lymphatic system agenesis that is typically bilateral, hypoplastic lymphatic drainage which is usually unilateral and rarely valvular insufficiency. Secondary causes consist of trauma, infection, filariasis, malignancy, surgery and radiotherapy (Sadeghi, 2010 \& Szuba, 1998). Regardless of the pathogenesis, it is most often a chronic, unrelenting condition, posing long-term physical and psychological difficulties for patients as well as a complex therapeutic challenge for clinicians (Szuba, 1998).

Imaging modalities for example ultrasonography, magnetic resonance imaging, computed tomography (CT) scan, lymphangiography and lymphography are utilised in evaluating lymphoedema. Previously, lymphangiography had been used to investigate lymphatic disorders, but it was time consuming, involved direct cannulation of lymph vessels and associated with complications such as infections and hypersensitivity (Lam, 2014). At present, lymphoscintigraphy has replaced lymphangiography as the gold standard and investigation of choice for lymphatics assessment (Yuan, 2006).

Lymphoscintigraphy commonly involves administration of small sized colloid particles usually around 50-70 nanometres tagged to low dose Technetium $99 \mathrm{~m}$ and subcutaneously injected into the lymphatic webs (Sadeghi, 2010). Gamma rays produced by this radiopharmaceutical can be then detected by gamma camera allowing visualisation or mapping of tracer drainage and lymph nodes localisation. Hence, lymphoscintigraphy has been described to be safe and effective method for evaluating suspected cases of lower limb lymphoedema with no reported complications (Lam, 2014 \& Yuan, 2006).

The expected normal scintigraphy pattern of lymphatic passage is symmetric movement of tracer in the limbs, discrete lymphatic channels and early visualisation of regional lymph nodes within 15-20 minutes (Nganga, 2018 \& Sadeghi, 2010). In contrast, recognised abnormal scan findings are non-visualisation or asymmetric visualisation of regional nodes, interrupted flow, dilated channels with collaterals, dermal backflow, decreased number of regional nodes and presence of popliteal nodes that indicate abnormal deep lymphatic flow (Kalawat, 2012; Nganga, 2018 \& Sadeghi, 2010).

Obesity was a common problem for the patients with chronic swollen legs in this case series. Although none had any lower limb trauma or documented deep vein thrombosis, the first patient has underlying liver cirrhosis whereas the other patients had history of cellulitis. All patients demonstrated abnormal lymphoscintigraphy features of lymphoedema bilaterally including dermal backflow and abnormal tracer progression. Bilateral popliteal lymph nodes were seen in a patient who initially presented with only a prominent right lower limb swelling.

Main differential diagnoses for bilateral lower limb oedema comprise of venous thrombosis, heart failure, liver failure, kidney disease or nephrotic syndrome, hypothyroid myxoedema and venous insufficiency (Hennessy, 2017 \& Shastri, 2008). It was highlighted that lymphoscintigraphy should be considered in the management algorithm for patient with chronic bilateral lower limb swelling (Hennessy, 2017). Lymphoscintigraphy would be able to help to differentiate other differential diagnoses of lower limb lymphoedema including obesity, chronic venous insufficiency and systemic diseases (Lam, 2014).

(Barral, 2013) conducted a retrospective study of 154 cases of lower limb lymphoscintigraphy and reported $30.5 \%$ had BMI over $30 \mathrm{~kg} / \mathrm{m} 2$. They found that 149 patients demonstrated some abnormality on lymphoscintigraphy and up to $40.3 \%$ had bilateral involvement (Barral, 2013). Obesity can cause lower limb lymphoedema with reciprocal relationship whereby obesity impairs lymphatic transport capacity and in turn the impaired lymphatic function promotes adipose deposition (Greene, 2015 \& Mehrara, 2014). Lymphoedema may develop as a result of cellulitis and similarly the presence of chronic lymphoedema predisposes patients to recurrent cellulitis, leading to an untoward vicious cycle (Al-Niaimi, 2009).

In brief, treatment of lymphoedema depends on its aetiology and usually includes non-surgical approach 
such as compression therapy and pharmacotherapy as well as surgical management for instance microsurgical intervention and anastomosis (Szuba, 1998). Although some cases particularly primary lymphoedema tend to be progressive and non-curable, treatment may provide relief or improvement in symptoms and signs (Heinig, 2017). Besides aiding the diagnosis and determine lymphoedema severity, lymphoscintigraphy may also assist to evaluate treatment response during follow-up (Sadeghi, 2010).

(Kalawat, 2012) reported that $83 \%$ of 24 patients with clinically positive leg swelling of unclear aetiology were found to be positive for lymphoedema on lymphoscintigraphy and exact localisation of inguinal nodes was able to be documented pre-operatively. Patients were then treated with decongestive lymphatic therapy. Subsequent lymphoscintigraphy in 9 cases who underwent nodo-venous shunt surgery demonstrated normal lymphatic channels with no recurrence suggesting the important role of this modality in monitoring the patients (Kalawat, 2012).

\section{CONCLUSION}

Lymph oedema is a chronic and progressive disease that cause swelling of tissue due to inadequate lymphatic function. Although it usually can be identified by history and physical examination, diagnostic confusion is common. In patients with chronic swollen legs who are suspected of having lymphoedema, lower limb lymphoscintigraphy is a simple and useful investigation tool for further evaluation. This article illustrated the usefulness of lymphoscintigraphy in evaluating cases of chronic swollen legs who eventually revealed scintigraphy findings suggestive of bilateral lymphoedema.

\section{Ethics}

This case report has been registered with the National Medical Research Register, Ministry of Health Malaysia (NMRR-19-906-48052) and received the permission from the Head of Nuclear Medicine Department, Hospital Kuala Lumpur. Efforts have been taken to ensure confidentiality of the patients. The author declares no conflict of interests and did not receive any fund or grant for this publication.

\section{ACKNOWLEDGEMENT}

Firstly, I would like to thank the Director General of
Health Malaysia for his permission to publish this article. I also like to acknowledge my dedicated colleagues, officers and staff at the Nuclear Medicine Department, Hospital Kuala Lumpur for their continuous support, encouragement and kind assistance.

\section{CONFLICT OF INTERESTS}

The authors declare that they have no conflict of interest.

\section{REFERENCES}

Al-Niaimi, F. \& Cox, N. (2009) Cellulitis and lymphoedema: A vicious cycle. Journal of Lymphoedema, 4(2) pp:38-42

Barral, C.M., Stehling, A.P., da Silva, A.C.M., Castro, A.C., Ivo, C.S., Korman, D.E., Couto, E.M., Magalhães, L.N., Carvalho, L.A., Félix, M.T.M., Machado, F.S. (2013) Lymphoscintigraphy of the lower limbs: A retrospective study of 154 cases from March 2009 to June 2010. Revista Médica de Minas Gerais, 23(2) pp: 182-192.

Greene, A.K., Grant, F.D. \& Maclellan, R.A. (2015) Obesity-induced Lymphedema Nonreversible following Massive Weight Loss. Revista Médica de Minas Gerais, 3(6): e426

Heinig, B., Lotti, T., Tchernev, G. \& Wollina, U. (2017) Hereditary Lymphedema of the Leg - A Case Report. Open Access Macedonian Journal of Medical Sciences, 5(4) pp:451-453.

Hennessy, M.M., O’Brien, G.C. (2017) Grossdependent lower limb lymphoedema. Clinical Case Reports, 5(2) pp: 150-153.

Kalawat, T.C., Chittoria, R.K., Reddy, P.K., Suneetha, B., Narayan, R. \& Ravi, P. (2012) Role of lymphoscintigraphy in diagnosis and management of patients with leg swelling of unclear etiology. Indian Journal of Nuclear Medicine, 27(4) pp:226-230.

Lam, M.C., Luk, W.H. \& Tse, K.H. (2014) Lymphoscintigraphy in the evaluation of lower extremity lymphedema: Local experience. Hong Kong Medical Journal, 20(2) pp:121-125.

Mehrara, B.J. \& Greene, A.K. (2014) Lymphedema and 
Obesity: Is There a Link? Plastic and Reconstructive Surgery., 134(1):154e-160e

. Nganga, E.C., Gitau, S. \& Makhdomi, K. (2018) Lower limb lymphoscintigraphy patterns among patients with lower limb lymphedema: A pictorial essay. Clinical and Translational Imaging, 6 pp:135-143.

Sadeghi, R., Kazemzadeh, G. \& Keshtgar, M. (2010) Diagnostic application of lymphoscintigraphy in the management of lymphoedema. Hellenic Journal of Nuclear Medicine, 13(1) pp: 6-10

Szuba, A. \& Rockson, S.G. (1998) Lymphedema: classification, diagnosis and therapy. Vascular Medicine, 3(2) pp:145-156

Yuan, Z., Chen, L., Luo, Q., Zhu, J., Lu, H. \& Zhu, R. (2006) The role of lymphoscintigraphy in extremity lymphedema. Annals of Nuclear Medicine, 20(5) pp:341-344 